\title{
Advantages of Crystallographic Fragment Screening: Functional and Mechanistic Insights from a Powerful Platform for Efficient Drug Discovery
}

Disha Patel, Joseph D. Bauman, and Eddy Arnold

Center for Advanced Biotechnology and Medicine, and Department of Chemistry and Chemical Biology, Rutgers University, Piscataway, NJ

\begin{abstract}
X-ray crystallography has been an under-appreciated screening tool for fragment-based drug discovery due to the perception of low throughput and technical difficulty. Investigators in industry and academia have overcome these challenges by taking advantage of key factors that contribute to a successful crystallographic screening campaign. Efficient cocktail design and soaking methodologies have evolved to maximize throughput while minimizing false positives/negatives. In addition, technical improvements at synchrotron beamlines have dramatically increased data collection rates thus enabling screening on a timescale comparable to other techniques. The combination of available resources and efficient experimental design has resulted in many successful crystallographic screening campaigns. The three-dimensional crystal structure of the bound fragment complexed to its target, a direct result of the screening effort, enables structure-based drug design while revealing insights regarding protein dynamics and function not readily obtained through other experimental approaches. Furthermore, this "chemical interrogation" of the target protein crystals can lead to the identification of useful reagents for improving diffraction resolution or compound solubility.
\end{abstract}

\section{Keywords}

X-ray crystallography, fragment screening, HIV, influenza

\section{Abbreviations}


BpGCDH B. pseudomallei glutaryl-CoA dehydrogenase, DHNA 7,8-dihydroneopterin aldolase, DMSO dimethylsulfoxide, FBDD fragment-based drug discovery, HCV hepatitis $\mathrm{C}$ virus, HIV human immunodeficiency virus, NMR nuclear magnetic resonance spectroscopy, NNRTI non-nucleoside reverse transcriptase inhibitor, $\mathrm{PA}_{\mathrm{N}}$ influenza endonuclease, PR protease, RT reverse transcriptase, TMAO trimethylamine-N-oxide, TS thymidylate synthase

\section{Introduction}

Fragment-based drug discovery (FBDD) has gained popularity as an effective and efficient approach for identifying pharmacophores for lead development. FBDD entails screening of small-molecule libraries against a target protein to identify weakly potent, bioactive molecules. The smaller, less complex nature of fragments increases the probability of binding to a target protein, resulting in higher hit rates and efficient search of diverse chemical space (Hadjuk et al., 2007; Hesterkamp et al., 2008; Erlanson et al., 2004). Hits identified from fragment screening do not require deconstruction and can be efficiently developed for specificity and inhibitory activity.

Today, an array of biophysical methods has been developed to rapidly identify weakly binding fragments to a target protein. Nuclear magnetic resonance (NMR) and X-ray crystallography were among the first applied and, more recently, surface plasmon resonance (SPR) and differential scanning fluorimetry (thermal shift) have become popular as primary screening methods. X-ray crystallography provides structural information that enables rapid and efficient assessment of hits with respect to synthetic tractability for structure-based drug design. With respect to FBDD, high-resolution Xray crystal structures provide a three-dimensional perspective of the binding landscape 
for potential fragment expansion, merging, or linking. Compared with other screening methods, X-ray crystallographic fragment screening is unmatched in terms of the range of ligand binding affinity (sub-nanomolar to millimolar, limited only by solubility of the ligand) and the size of the target macromolecule (up to megadaltons, limited only by crystal robustness and resolution limit). However, application of X-ray crystallography as a primary screening method has been under-appreciated and often considered to be impractical due to its relatively low throughput and highly resource-intensive nature.

Recent advances in crystal transport, robotic crystal mounting, powerful detectors, and automated data collection software have significantly improved the throughput and reduced the amount of labor associated with this approach. The technical improvements combined with an efficient strategy for data collection and rapid hit identification makes X-ray crystallography an attractive approach for primary screening, in that it not only identifies fragment binding but also provides a three-dimensional structure that can enable and facilitate rapid structure-based optimization.

\section{Historical Perspective}

Mattos and Ringe (1996) were among the first to demonstrate the potential for systematic exploration of potential small molecule binding sites by soaking organic solvents into crystals that diffract X-rays to high resolution. This approach, referred to as multiple solvent crystallographic structures (MSCS), uses organic solvents as probes for locating and characterizing potential binding sites for ligand design (Allen et al., 1996; Mattos and Ringe, 1996; English et al., 1999). Superimposing crystal structures from soaking with different organic solvents can provide insight regarding the conformational landscape, chemical complementarity, plasticity, and surface hydration for a binding site 
of interest. In addition, the structural information, with the aid of molecular modeling, can be used to develop pharmacophoric models or improve binding affinity and specificity for lead optimization (Mattos et al., 2006; Behnen et al., 2012). However, difficulties in reliably predicting optimal binding modes and overcoming exquisitely demanding stereochemical requirements for linked molecules have limited the application of MSCS for drug discovery.

In 1998, Stout et al. developed a deconstruction approach using E. coli thymidylate synthase (TS) and its natural substrate, deoxyuridine monophosphate (dUMP), to understand ligand binding. Structural analysis of small molecule fragments of dUMP in complex with TS revealed the relative contributions of each individual chemical component to the overall binding of dUMP. This modular approach to deconvolution of a ligand hinted that the opposite methodology where compatible fragments can be merged in an additive manner might be promising.

Christophe Verlinde and Wim Hol were among the first to utilize X-ray crystallography to identify small molecule binding from a fragment library of 384 compounds to triose phosphate isomerase from Trypanosoma brucei (Verlinde et al., 1997). For high-throughput purposes, crystallographic screening was conducted by soaking pre-formed crystals into three mixtures, commonly referred to as cocktails, of 128 compounds (Verlinde et al., 2009). Electron density from one cocktail soak indicated fragment binding; however, compound identification was not readily possible due to a limited resolution of $2.8 \AA$. Subsequent deconvolution indicated that the observed electron density was likely from a product of a chemical reaction between fragments within the cocktail. Based on the experimental results, it was clear that X-ray 
crystallography in combination with cocktails could be used for fragment hit identification. However, design of these cocktails should take into consideration chemical reactivity, solubility, and shape diversity.

Initial studies conducted by both academic and industrial groups pioneered the use of X-ray crystallography as a primary screening method for fragment-based drug discovery. Along the way, several important experimental factors were found to be critical for a successful crystallographic screening campaign. This review will describe some of the experimental challenges and approaches associated with X-ray crystallographic fragment screening. In addition, interesting biological and technical insights gained through crystallographic fragment screening campaigns will also be highlighted.

\section{Experimental Considerations}

\subsection{Cocktail Design}

The approach to cocktail crystallography devised by Nienaber and colleagues (2000) at Abbott Laboratories led to the development of the CrystaLEADS system. To enable rapid hit identification, cocktails were designed to maximize the structural diversity of the fragments. Urokinase was used to demonstrate the applicability of CrystaLEADS for hit identification. Nine crystals of urokinase were used to screen a fragment library of 61 compounds grouped into cocktails of 6-8 fragments. Screening identified five promising fragments hits, of which 8-hydroxy-2-aminoquinoline was found to be the most potent. With the availability of a crystal structure combined with previously established SAR, a single modification of the fragment hit allowed for extension into an adjacent pocket leading to a 151-fold improvement in potency. More 
commonly, the CrystaLEADS approach entails grouping a 10,000 compound library into 100 fragments per cocktail. The application of this cocktail design was demonstrated by crystallographic screen of 7,8-dihydroneopterin aldolase (DHNA). Screening identified two fragment hits, which were subsequently developed into lead compounds with $\mu \mathrm{M}$ activity against DHNA (Sanders et al., 2004).

Astex Pharmecuticals pioneered a successful approach for cocktail crystallography as part of their proprietary Pyramid platform. Cocktail design limited the number of fragments per cocktail to four and strongly emphasized chemical diversity within a cocktail (Hartshorn et al., 2005). This allowed for efficient deconvolution and reduced the likelihood of multiple fragment binding. In contrast, Johnson and Johnson's approach to cocktail design focused on designing cocktails of five compounds with similar shape (Spurlino, 2011). This approach took advantage of multiple-fragment binding within one cocktail to strengthen the electron density for a fragment hit.

The Biomolecular Structure Center at University of Washington as part of the Medical Structural Genomics for Protozoan Parasites Consortium (MSGPPC) reported using a computational approach for cocktail design. A 680 compound library was designed from an initial library of 9,486 compounds using a series of filters to limit the size, number of heteroatoms, hydrophobicity, and number of rotatable bonds as well as to remove compounds that were chemically reactive and expensive. A shape fingerprint analysis was conducted to design 68 cocktails of 10 structurally diverse compounds. This not only takes advantage of shape diversity to facilitate deconvolution but also allows for rapid screening.

\subsection{Protein crystallization}


Verlinde et al. (2009) described the importance of protein crystallization towards the success of a fragment screening campaign. The MSGPPC screened a total of 26 protein targets, of which 19 were found to be impervious to fragment binding. The high percentage of nonproductive targets was due to poor resolution (lower than $2.8 \AA$ ), reduced crystal stability or robustness in the soaking conditions, or simply no observed fragment binding. The number of cocktails that could be successfully screened was limited by either poor resolution or crystal robustness for certain protein targets. For instance, only 42 useful datasets were obtained from soaking 66 cocktails into 147 crystals of Leishmania major coproporphyrinogen III oxidase. Similarly, only 42 of the 68 cocktails screened against Leishmania naiffi uracil-DNA glycosylase produced datasets useful for fragment identification.

Thus, the success and throughput of crystallographic fragment screening is heavily dependent on resolution and robustness of the protein crystal. Ideally, protein crystals that diffract X-rays to high resolution (better than $2.5 \AA$ ) help to reduce ambiguities that may occur upon ligand binding. Crystal engineering and proteolytically stable protein constructs can be used to improve resolution for proteins that are either highly flexible or poorly diffracting. Crystal robustness in a high concentration fragment soaking solution is essential as it allows for rapid screening and reduces the amount of labor associated with this approach. Either alternative crystal forms or co-crystallization will be necessary if the crystals are either too fragile to handle or behave poorly during soaking.

Prior to conducting a fragment screening campaign for HIV-1 reverse transcriptase (RT), crystal engineering was used to improve the resolution quality and 
reproducibility of protein crystals by introducing point mutations and C-terminal truncations to reduce the surface entropy of the protein (Bauman et al., 2008). Despite an improvement in resolution, crystal robustness proved to be a major challenge during our fragment screening campaign against HIV-1 RT (Bauman et al., 2013a). Originally, a high-resolution construct of the apo HIV-1 RT (RT69A) was selected for screening. However, the RT69A crystals were not able to withstand the soaking conditions, resulting in a decrease in the throughput of the method. The high sensitivity of these crystals was speculated to be a consequence of fragment binding to the allosteric non-nucleoside reverse transcriptase inhibitor (NNRTI) binding pocket. The formation of this binding site requires large conformational changes, resulting in collapse of the crystal lattice. Of the crystals that survived the cocktail soaks, only one out of ten crystals diffracted to useful resolution. To improve throughput and data collection, an alternate protein construct, RT52A, in complex with a potent NNRTI, rilpivirine, was used to block access to the NNRTI-binding pocket and limit protein flexibility. These crystals were extremely robust with $\sim 90 \%$ of crystals surviving cocktail soaks and maintaining high quality X-ray diffraction (1.8-2.0 ̊ resolution).

It is necessary for the protein in the selected crystal form to be in a biologically relevant conformation and binding sites of interest should not be occluded by proteinprotein crystal contacts. This helps to maintain a correlation with the enzymatic assays that are commonly used to characterize the inhibitory activity of the fragment hits. Prior to conducting a fragment screening campaign against the influenza A endonuclease $\left(\mathrm{PA}_{\mathrm{N}}\right)$, it was necessary to identify a new crystal form because the two published crystal forms were not useful for structure-based drug design. One crystal form was very 
difficult to reproduce while other published structure of $\mathrm{PA}_{\mathrm{N}}$ had an active site occluded by a crystallographically symmetric copy of the protein (Dias et al., 2009; Yuan et al., 2009; Bauman et al., 2013b). Additionally, binding sites should not be occupied by chemical components from the crystallization condition. This can result in false negatives since the fragments, at a lower concentration, are competing with the crystallization components for binding.

\subsection{Optimization of Fragment Soaking}

Crystallographic fragment screening is typically conducted at high compound concentrations (10 $\mathrm{mM}$ and higher) to ensure full occupancy for low-affinity ligands. The most common method for screening entails soaking pre-formed crystals into a solution of fragment(s) for a period of time, which can range from as short as several seconds to days. To ensure crystal stability and longevity during the soak, diluting the fragment or cocktail using the crystallization buffer is necessary. However, this may come at the expense of fragment solubility and therefore may limit the concentration of fragment available for binding. Unlike most biophysical methods, it is not required for the fragment to be fully soluble at the tested concentration as it is possible to recover crystals from soaking drops that are only partially transparent due to precipitation. During the screening campaign against HIV-1 RT, fragment solubility in the crystallization buffer was improved using L-arginine as an additive (Bauman et al., 2013a). Initial soaking experiments revealed electron density for some hydrophobic fragments only in the presence of L-arginine. We speculate that this improvement is most likely due to the guanidinium functionality of L-arginine, which can form hydrogen bonding and/or $\pi$-stacking interactions (Flocco et al., 1994). 
The solvent used for solubilizing fragments can have a tremendous impact on crystal stability during soaking. For instance, many protein crystals can only tolerate up to $10 \%(\mathrm{v} / \mathrm{v})$ dimethylsulfoxide (DMSO), a commonly used solvent for fragment solutions. Soaking at higher concentrations of DMSO can either cause the crystal lattice to collapse or have a detrimental effect on X-ray diffraction. Incorporating DMSO into the protein crystallization condition can circumvent sensitivity of protein crystals to DMSO (Perryman et al., 2010). The cryoprotective properties of DMSO (Atwell et al., 2010) can be advantageous as it allows for a single solution for fragment soaking and cryoprotection (Bauman et al., 2013 a,b). This minimizes crystal damage by mechanical stress and reduces the amount of time needed for soaking and freezing.

The solvent, due to its higher concentration, may also bind throughout the protein. Initial soaking experiments during the HIV-1 RT fragment screening campaign revealed electron density for DMSO molecules throughout the protein regardless of which cocktail was soaked. A crystal structure obtained from a soak with DMSO without any fragment present revealed 16 binding sites throughout HIV-1 RT (Figure 1). This structure proved to be valuable as a blank reference to avoid misinterpretation of solvent binding as fragment binding (Bauman et al., 2013a). In the event that DMSO should significantly affect crystal stability or fragment binding, an alternate solvent, such as water or methanol, can be used for solubilizing fragments.

The crystallization condition itself can prove to be problematic for soaking by altering the fragment solubility in solution. Davies et al. (2012) described a fragment screening campaign against a proprietary target PRP4 that was productive but failed to identify carboxylic acid-containing fragments, which were predicted to have activity in 
an independent assay. Lack of binding for these fragments, was attributed to $200 \mathrm{mM}$ calcium chloride present in the crystallization condition. Specifically, the calcium ion was believed to reduce the solubility of the acid containing fragments and thereby prevent their binding. Replacing the calcium chloride with an alternate chloride salt in the soaking solution enabled fragment binding to be visualized. Thus, optimization of the soaking conditions to ensure protein stability, fragment solubility, and technical ease is a necessary pre-requisite prior to initiating a crystallographic fragment-screening campaign.

\subsection{Fragment Screening by Co-crystallization}

An alternative approach for fragment screening is the co-crystallization of the protein in the presence of the fragment. This method is resource-intensive since each compound requires a separate crystallization experiment. Thus, co-crystallization is commonly used when fragment soaking either affects crystal stability or fails to identify any hits. Co-crystallization with fragment cocktails can be challenging due to issues with fragment solubility and long-term stability. Furthermore, ligand binding can alter the mobility of the protein thus requiring a change in the crystallization condition. This approach cannot identify weak fragment binders since high concentration of fragments can interfere with crystallization. Perryman et al. (2010) used co-crystallization approach to identify fragment binding to HIV-1 protease after soaking individual fragments into two different crystal forms failed. Co-crystallization screening at 10 and $2.5 \mathrm{mM}$ fragment concentrations using a crystallization condition for a $\mathrm{P} 6_{1} 22$ crystal form of HIV-1 protease produced two new crystal forms, resulting in the identification of three fragment hits.

\subsection{Quality Control}


Quality control is often considered as an afterthought mainly because the fragments purchased are of nominally high purity. However, it is possible that the silent impurities, such as metal ions, synthetic byproducts, or products of compound degradation, can produce false positives, which can often result in a tremendous waste of time and resources. The high fragment concentrations used in crystallographic screening can exacerbate these issues because even minor contaminants can be present at sufficient concentrations leading to significant electron density changes and false positives.

During the screening campaign against HIV-1 RT, electron density for binding of a commercially obtained fragment, $\mathbf{1}$, was observed; however, fitting the compound into the density proved to be challenging (Figure 2A). Promising RNase H inhibitory activity in the enzymatic assays for " 1 " fueled excitement, however, subsequent crystallization experiments by both soaking and co-crystallization failed to demonstrate sensible electron density for this fragment. The next sample of " 1 " purchased showed a dramatic loss of inhibitory activity and difference electron density. Subsequent crystallographic analyses using anomalous difference maps indicated that the observed electron density was unexpectedly due to contaminating metal ions (Figure 2B). The inhibition was most likely by competition of the contaminating metal with $\mathrm{Mg}^{2+}$ ions used in the RNase $\mathrm{H}$ assay. Our and others' (often anecdotal) experiences suggest that compound quality control can vary widely among commercial vendors. Given the frequent waste of considerable time, effort, and resources pursuing contaminants, rigorous quality control of fragments and cocktails should be an integral component of all fragment-screening platforms.

\subsection{High-Throughput Crystallography}


Application of X-ray crystallography as a primary method for fragment screening can be limited by the lack of high throughput and its highly specialized nature. However, recent technological improvements in crystal freezing and synchrotron resources have significantly reduced these hurdles. For our screening campaigns, use of a cassette or puck system facilitated the transport of larger quantities of crystals to the synchrotron. A total of seven ALS-style pucks with each puck holding 16 crystals can be transported in a CP100 Dewar. This allowed for the transport of 112 crystals per dewar in comparison to only 56 crystals using the traditional cane transport system. The pucks allowed for rapid freezing of the crystals and reduced handling error compared to the cane system. Additionally, use of the puck system significantly reduced ice buildup around the crystals, thus reducing the time spent at the beam rinsing the crystal with liquid nitrogen to remove the ice. The compatibility of puck systems with the robotic crystal automounters available at synchrotrons further facilitated rapid data collection by reducing the time, labor, and the risk to the samples associated with manual mounting and dismounting a crystal. Rapid identification of potential problems with the automounter system is critical because malfunctions can lead to crystal loss through temperature fluctuations and frosting. These problems, though common in the early days of these systems, are now rare and are more than compensated for by the extraordinary increases in throughput and reliability enabled by this instrumentation.

Taking advantage of beamline resources, we developed a high-speed data collection strategy aimed at maximizing data collection and enabling rapid hit identification. Initial screening consisted of low-resolution data collection by limiting the exposure time and increasing the oscillation range of the frames. This reduced the 
duration of dataset collection with a minimal impact on the diffraction resolution of the crystals. Fragment binding was determined from a visual inspection of difference Fourier maps with $\mathrm{F}_{\mathrm{o}}-\mathrm{F}_{\mathrm{o}}$ coefficients derived from subtracting the structure factor magnitudes of the DMSO reference dataset from those of the fragment/cocktail soaked crystal. An advantage of the $\mathrm{F}_{\mathrm{o}}-\mathrm{F}_{\mathrm{o}}$ difference map is that phases from the reference structure can be used without requiring refinement of the fragment-containing dataset. Crystals with changes in the electron density due to fragment binding and/or repositioning of protein residues or solvent molecules were prioritized for further studies. Although this can be done through standard refinement software such as CCP4 (Winn et al., 2011) and PHENIX (Adams et al., 2010), specialized software, such as AutoSolve (developed at Astex), has been developed to automate this process (Blundell et al., 2002). Crystals for which fragment binding was detected were remounted and subjected to high-resolution data collection. Both the high and low-resolution datasets were then merged to improve the electron density for the bound fragment for subsequent refinement and analysis.

Advancements in detector technology have also contributed to the improvement in efficiency and throughput of X-ray crystallography as a screening method. The ultrarapid readout and high quantum efficiency associated with the Pilatus 6M detector allows for continuous crystal rotation without opening and closing of the shutter after each frame (Henrich et al., 2009). The Pilatus 6M detector reduced the average time for highresolution data collection for an influenza endonuclease crystal from six to two minutes. When combined with an automounter, we were able to collect 15 datasets per hour with approximately half of the time used for mounting/unmounting and crystal centering. As a result, complete datasets for a total of 112 influenza endonuclease crystals were collected 
within a 12-hour X-ray beam allocation. Due to the rapid data collection with the Pilatus detector, it was no longer necessary to utilize the high-speed data collection strategy previously described. These improvements in data collection and processing have dramatically reduced the difficulties commonly associated with crystallographic fragment screening.

\section{Biological Applications of Fragment Screening}

\subsection{Probing Protein Dynamics}

Despite the constrained environment within a crystal lattice, proteins and other macromolecules still possess significant degrees of flexibility, which can be investigated through crystallographic fragment screening. The extent of protein dynamics within a crystal depends on the intrinsic conformational flexibility of the protein target, the presence of bound ligands, the crystal form under investigation, and conditions including temperature, $\mathrm{pH}$, and buffer and salt concentrations. The HIV-1 RT screening campaign revealed not only fragment binding to existing crevices but also successfully trapped previously unseen protein conformations. In particular, fragment binding to the Knuckles pocket, which is normally a buried cavity in previously reported RT structures, was successfully characterized despite a $1.2 \AA$ backbone shift in the fingers subdomains (Figure 3). Time-based soaking experiments ranging from 10 seconds to 30 minutes revealed fragments binding to the Knuckles, demonstrating the dynamic nature of RT in crystallo. Visualization of these dynamically accessible states is possible when fragment binding stabilizes a transient protein conformation. Similar observations were made from a crystallographic screening campaign targeting glutaryl-CoA dehydrogenase from $B$.

pseudomallei (BpGCDH) (Begley et al., 2011). Fragment binding to the apo BpGCDH 
induces a conformational change in which the binding site for FAD, a co-factor, becomes occupied with protein residues. Presumably, the fragment can inhibit the enzyme through stabilization of an alternative co-factor pocket conformation.

Probing protein dynamics using fragments is not limited to small-scale, transient conformations, larger domain interactions can also be explored. Saalau-Bethell and colleagues (2012) at Astex Therapeutics altered the equilibrium between the active and inactive conformations of hepatitis C virus (HCV) NS3-NS4A protein. Crystallographic screening identified fragment binding to a new allosteric site located between the protease and helicase domains. Crystal structure of a chemical lead developed from the original hit bound to HCV NS3-NS4A suggests that compound binding to the allosteric site stabilizes the closed conformation, thereby preventing the domain movement necessary to form the active conformation, which results in a loss of protease activity necessary for $\mathrm{HCV}$ polyprotein processing.

\subsection{Functional Studies}

Fragments are not solely limited to probing protein flexibility but can also enable a better understanding of enzymatic function. Two of the eight fragments identified from a crystallographic fragment screening against influenza $\mathrm{PA}_{\mathrm{N}}$ revealed a previously undetected third metal ion within the endonuclease active site (Bauman et al., 2013b). This was unexpected as a two metal ion-binding model had been established based on previous structural and calorimetric studies. To determine whether this third metal binding was simply a non-biologically relevant artifact of fragment binding, crystals of apo EDTA-treated $\mathrm{PA}_{\mathrm{N}}$ were soaked with a range of metal ions $\left(\mathrm{Mn}^{2+}, \mathrm{Zn}^{2+}, \mathrm{Ca}^{2+}\right.$, and $\mathrm{Ni}^{2+}$ ) at high concentrations. Of the metals soaked, the $\mathrm{Ca}^{2+}$ soak revealed strong electron 
density for the third metal (Figure 4) in the absence of fragment. Based on structural studies with related endonucleases, we hypothesize that this third metal ion may stabilize the negatively charged transition state during catalysis (Horton et al., 2004). Thus, this third metal ion binding to the endonuclease active site is not simply a by-product of fragment binding and warrants further investigation to fully understand its functional and mechanistic relevance.

Small-molecule fragments can also be used to characterize the enzymatic function of unknown proteins as demonstrated by Shumilin et al. (2012). The natural substrates for two unknown proteins, YjeF_N and PF01256, were identified from a crystallographic screening of a small molecule library of metabolites followed by a focused screen of structurally similar molecules. By visualizing metabolites, reactants or reaction products bound directly in the enzyme active site, specific follow-up experiments could be prioritized to confirm the putative enzymatic function of the protein. Thus, fragment screening by X-ray crystallography has proven to be a powerful tool for probing the biology of enzyme structure and function.

\section{Technical Insights}

\subsection{Halogens}

Halogenated compounds are often under-represented in fragment libraries due to the arbitrary molecular mass cutoffs. However, these fragments are highly versatile allowing for rapid hit identification, specifically anomalous X-ray diffraction (Blaney et al., 2006) and isotope enrichment for NMR spectroscopy (Dalvit et al., 2002) can be used to detect binding of brominated and fluorinated fragments, respectively. Furthermore, halogenated fragments can serve as molecular probes for binding through favorable 
halogen-bonding interactions. Wilcken et al. (2012) assembled and screened a halogenenriched fragment library to take advantage of predicted halogen-bonding interactions based on docking studies to develop small molecule stabilizers of a mutant Y220C p53.

Analysis of fragment hits from the HIV-1 RT fragment screening campaign revealed a preference for halogenated fragments. Specifically, seven out of 29 fluorinated fragments and four out of 17 brominated fragments screened were identified as hits. The higher hit rates for halogenated fragments indicated that a halogen-focused fragment library could be beneficial.

Based on the HIV-1 RT results, Tiefenbrunn et al. (2013) demonstrated that a brominated library could be useful in targeting recalcitrant crystal forms, which have yielded low hit rates during screening. A library of 68 brominated fragments was screened to identify binding to two sites of interest - exosite and flap site-within HIV protease. At least one bromine anomalous peak was detected for 23 of the 68 compounds. However, most of these compounds were not of interest as either electron density for only the bromine atom or binding to interstitial or the TL-3 sites was observed. Clear binding was observed for two fragments - each binding to the exosite and flap siteyielding a hit rate of $3 \%$. The hit rate increased to $6 \%$ if compound binding at the TL-3 site is taken into account. Compared to the initial ActiveSite screen (Tiefenbrunn et al., 2013), a four- to eight-fold improvement in hit rate was observed for the brominated library. Thus, the focused brominated fragment library proved to be valuable not only for hit identification, but also for improving hit rates, presumably through halogen-bonding interactions.

\subsection{Trimethylamine-N-oxide: A Useful Cryocrystallography Additive}


An unexpected observation from soaking crystals of the HIV-1 RT/rilpivirine with a wide variety of solutions was the identification of trimethylamine- $\mathrm{N}$-oxide (TMAO) as a useful reagent for improving the diffraction quality of crystals. Soaking at a concentration of $6 \%(\mathrm{w} / \mathrm{v})$ TMAO improved the resolution of the HIV-1 RT crystals from $1.80 \AA$ to $1.51 \AA$ (Kuroda et al., 2013). A similar improvement in diffraction resolution with TMAO was also observed for crystals of influenza endonuclease and other HIV-1 RT complexes. Inspired by our observations, crystals of alphavirus polyprotein were treated with $5 \%(\mathrm{w} / \mathrm{v})$ TMAO during cryoprotection, resulting in a similar improvement in resolution (Shin et al., 2012). Improvement in diffraction resolution for both the HIV-1 RT and alphavirus polyprotein crystals was observed solely through soaking of TMAO, not through co-crystallization.

TMAO has been explored previously as a useful biological or crystallographic reagent. At very high concentrations (around $4 \mathrm{M}$ ), TMAO by itself can act as an excellent cryoprotective agent (Mueller-Dieckmann et al., 2011). Biological studies have demonstrated that TMAO can improve protein folding and stability. Specifically, TMAO destabilizes unfavored protein conformations (Yancey and Somero, 1979; Yancey et al., 1982; Lin et al., 1994; Wang et al., 1997). Molecular dynamics simulations indicate that TMAO stabilizes the protein structure in solution by enhancing favorable water interactions (Zou et al., 2002). Thus, we hypothesize that the improvement in resolution quality from TMAO soaking is due to stabilization of the protein within the crystal lattice in a manner similar to dehydration of the crystals without any significant changes in the unit cell dimensions. 
A fortuitous benefit of the high-resolution crystal structure of HIV-1 RT in complex with rilpivirine was the discovery and clear assignment of a water molecule that makes hydrogen-bonding interactions with the cinnamonitrile group of rilpivirine (Figure 5). Through collaboration with the lab of Robin Hochstrasser, we identified this hydrogen bond to be an important contributing factor for the observed potency of rilpivirine using a combination of two-dimensional vibrational echo spectroscopy and molecular dynamics (Kuroda et al., 2013). The location of this water molecule as initially predicted by the molecular dynamics simulation was not concordant with the $1.80 \AA$ crystal structure of HIV-1 RT in complex with rilpivirine. However, the $1.51 \AA$ resolution crystal structure resulting from the TMAO soak revealed electron density for this water molecule, thus confirming the results from $2 \mathrm{D}$-IR and molecular dynamics studies.

Although TMAO improved X-ray diffraction quality of crystals, fragment soaking with TMAO as an additive proved to be challenging. Previously identified fragment hits from both HIV-1 RT and influenza $\mathrm{PA}_{\mathrm{N}}$ screening campaigns no longer bound in the presence of $6 \%(\mathrm{w} / \mathrm{v})$ TMAO. This may be due to a reduction in fragment solubility in the presence of TMAO, consistent with observation of precipitate formation in fragment/cocktail soaking solutions.

\subsection{Multiple Binding}

Fragment screening campaigns using biophysical screening methods, such as NMR and SPR, favor single molecule binding to a target protein and discard multiple binders as non-specific. When multiple binders are detected, aggregation rather than specific interactions is usually assumed to be responsible for the observed binding. 
However, it is possible for viable fragment hits to be discarded as false positives. X-ray crystallography, to a certain degree, can reduce this occurrence by facilitating rapid determination of molecular specificity. Structural characterization of a promiscuous fragment bound to multiple sites on a target protein can guide compound derivatization to improve specificity and potency. This was successfully demonstrated by the development of a new class of influenza endonuclease $\left(\mathrm{PA}_{\mathrm{N}}\right)$ inhibitors (Bauman et al., 2013b). A fragment hit, 5-chloro-3-hydroxypyridin-2(1H)-one, identified as binding to three separate sites, was pursued for structure-based drug design despite its multiple binding modes (Figure 6). Docking using the available crystal structure allowed for rapid and efficient fragment expansion to improve specificity and potency. Further optimization produced a single-binding-mode inhibitor of $\mathrm{PA}_{\mathrm{N}}$ with significant antiviral activity.

\section{Conclusion}

When suitable crystals of the target protein are obtainable, fragment screening by X-ray crystallography has been established to be a very powerful tool for drug discovery and development. In addition, it can also provide exceptional insights into functional aspects of the target protein. Crystallographic fragment screening has been successful in stabilizing previously unseen protein conformations as well as discovery of functional aspects of enzymes. The continual development of high-throughput crystallography and its unique benefits for structure-based drug design make crystallographic fragment screening an excellent tool for characterizing a protein and its interactions.

\section{Acknowledgements}


Data collection for unpublished work presented here was conducted at the Cornell High Energy Synchrotron Source (CHESS) and the National Synchrotron Light Source (NSLS) at Brookhaven National Laboratory. CHESS is supported by the NSF \& NIH/NIGMS via NSF award DMR-0225180, and the MacCHESS resource is supported by NIH/NCRR award RR-01646. Use of NSLS is supported by the U.S. Department of Energy, Office of Science, Office of Basic Energy Sciences, under Contract No. DE-AC02-98CH10886. We thank Michael Parniak (University of Pittsburgh) for the RNase H inhibition assay for compound 1. We are also thankful to Kalyan Das and other members of the Arnold laboratory for helpful discussions and inspiration for the figures. We are extremely grateful to NIH MERIT Award R37AI027690 and grant P50GM103368 for generous support of these efforts.

\section{References}

Adams, P. D., Afonine, P. V., Bunkóczi, G., Chen, V. B., Davis, I. W., Echols, N., Headd, J. J., Hung, L. W., Kapral, G J., Grosse-Kunstleve, R. W., McCoy, A. J., Moriarty, N. W., Oeffner, R., Read, R. J., Richardson, D. C., Richardson, J. S., Terwilliger, T. C., Zwart, P. H. (2010) PHENIX: a comprehensive Python-based system for macromolecular structure solution. Acta Crystallographica Section D Biological Crystallography, 66, 213-221.

Allen, K. N., Bellamacina, C. R., Ding, X., Jeffery, C. J., Mattos, C., Petsko, G. A., Ringe, D. (1996). An experimental approach to mapping the binding surfaces of crystalline proteins. Journal of Physical Chemistry, 100, 2605-2611.

Atwell, S., Brouillette, C. G., Conners, K., Emtage, S., Gheyi, T., Guggino, W. B., et al. (2010). Structures of a minimal human CFTR first nucleotide-binding domain as a monomer, head-to-tail homodimer, and pathogenic mutant. Protein Engineering, Design \& Selection, 23, 375-384.

Bauman, J. D., Das, K., Ho, W. C, Baweja, M., Himmel, D. M., Clark, A. D., Oren, D. A., Boyer, P. L., Hughes, S. H., Shatkin, A. J., Arnold, E. (2008) Crystal engineering of HIV-1 reverse transcriptase for structure-based drug design. Nucleic Acids Research, 36, 5083-5092.

Bauman, J. D., Patel, D., Baker, S., Vijayan, R. S. K., Xiang, A., Parhi, A., MartinezSobrido, L., LaVoie, E. J., Das, K., Arnold, E. (2013) Crystallographic fragment 
screening and structure-based optimization yields a new class of influenza endonuclease inhibitors. ACS Chemical Biology, 8, 2501-2508.

Bauman, J., Patel, D., Dharia, C., Fromer, M. W., Ahmed, S., Frenkel, Y., Vijayan, R. S. K., Eck, T. J., Ho, W. C., Das, K., Shatkin, A. J., Arnold, E. (2013) Detecting allosteric sites of HIV-1 reverse transcriptase by X-ray crystallographic fragment screening. Journal of Medicinal Chemistry, 56, 2738-2746.

Begley, D. W., Davies, D. R., Hartley, R. C., Hewitt, S. N., Rychel, A. L., Myler, P. J., Van Voorhis, W. C., Staker, B. L., Stewart, L. J. (2011) Probing conformation stats of glutaryl-CoA dehydrogenase by fragment screening. Acta Crystallographic Section F, 67, 1060-1069.

Behnen, J., Köster, H., Neudert, G., Craan, T., Heine, A., Klebe, G. (2012) Experimental and computational active site mapping as a starting point to fragment-based lead discovery. ChemMedChem, 7, 248-261.

Blaney J., Nienaber V., Burley S. K. Fragment-based lead discovery and optimisation using $\mathrm{X}$ - ray crystallography, computational chemistry, and high-throughput organic synthesis. W. Jahnke, D. A. Erlanson (Eds.), Fragment-based Approaches in Drug Discovery. Methods and Principles in Medicinal Chemistry, (2006) Wiley-VCH: Weinheim, Germany, 215-248.

Blundell, T.L., Jhoti, H. and Abell, C. (2002) High-throughput crystallography for lead discovery in drug design. Nature Reviews Drug Discovery, 1, 45-54.

Dalvit, C., Flocco, M., Veronesi, M., Stockman, B. J. (2002) Fluorine-NMR competition binding experiments for high-throughput screening of large compound mixtures. Combinatorial Chemistry High Throughput Screening, 5, 605-611.

Davies T. G., Tickle I. J. (2012) Fragment screening using X-ray crystallography. Topics in Current Chemistry, 317, 33-59.

Dias, A., Bouvier, D., Crepin, T., McCarthy, A. A., Hart, D. J., Baudin, F., Cusack, S., and Ruigrok, R. W. (2009) The cap-snatching endonuclease of influenza virus polymerase resides in the PA subunit. Nature, 458, 914-918.

English, A. C., Done, S. H., Caves, L. S., Groom, C. R., Hubbard, R. E. (1999) Locating interaction sites on proteins: the crystal structure of thermolysin soaked in $2 \%$ to $100 \%$ isopropanol. Proteins: Structure, Function, and Genetics, 37, 628-640.

Erlanson D. A., Wells J. A., Braisted A. C. (2004) Tethering: fragment-based drug discovery. Annual Review of Biophysics Biomolecular Structure, 33, 199-223.

Flocco, M. M., Mowbray, S. L. (1994) Planar stacking interactions of arginine and aromatic-side chains in proteins. Journal of Molecular Biology, 235, 709-717. 
Hadjuk P.J., Greer J. (2007) A decade of fragment-based drug design: strategic advances and lessons learned. Nature Reviews Drug Discovery, 6, 211-219.

Hartshorn, M. J., Murray, C. W., Cleasby, A., Frederickson, M., Tickle, I. J., Jhoti, H. (2005) Fragment-based lead discovery using X-ray crystallography. Journal of Medicinal Chemistry, 48, 403-413.

Henrich, B., Bergamaschi, A., Broennimann, C., Dinapoli, R., Eikenberry, E. F., Johnson, I., Kobas, M., Kraft, P. Mozzanica, A., Schmitt, B. (2009) PILATUS: A single photon counting pixel detector for X-ray applications. Nuclear Instruments and Methods in Physics Research Section A, 607, 247-249.

Hesterkamp T., Whittaker M. (2008) Fragment-based activity space: smaller is better. Current Opinion in Chemical Biology, 12, 260-268.

Horton, N. C., Perona, J. J. (2004) DNA cleavage by EcoRV endonuclease: two metal ions in three metal ion binding sites. Biochemistry, 43, 6841-6857.

Kuroda, D. G., Bauman, J. D., Challa, R., Patel, D., Troxler, T., Das, K., Arnold, E., Hochstrasser, R. M. (2013) Snapshot of the equilibrium dynamics of a drug bound to HIV-1 reverse transcriptase. Nature Chemistry, 5, 174-181.

Lin, T. Y., Timasheff, S. N. (1994) Why do some organisms use a urea-methylamine mixture as osmolyte? Thermodynamic compensation of urea and trimethylamine $\mathrm{N}$-oxide interactions with protein. Biochemistry, 33, 12695-12701.

Mattos, C., Bellamacina, C. R., Peisach, E., Pereira, A., Vitkup, D., Petsko, G. A., Ringe, D. (2006) Multiple solvent crystal structures: probing binding sites, plasticity, and hydration. Journal of Molecular Biology, 57, 1471-1482.

Mattos, C., Ringe, D. (1996) Locating and characterizing binding sites on proteins. Nature Biotechnology, 14, 595-599.

Mueller-Dieckmann, C., Kauffmann, B., Weiss, M. S. (2011) Trimethylamine N-oxide as a versatile cryoprotective agent in macromolecular crystallography. Journal of Applied Crystallography, 44, 433-436.

Nienaber, V. L., Richardson, P. L., Klighofer, V., Bouska, J. J. Giranda, V. L., Greer, J. (2000) Discovering novel ligands for macromolecules using X-ray crystallographic screening. Nature Biotechnology, 18, 1105-1108.

Perryman, A.L., Zhang Q., Soutter, H. H., Rosenfeld, R., McRee, D. E., Olson, A. J., Elder, J. E., Stout, C. D. (2010) Fragment-based screen against HIV protease. Chemical Biology Drug Design, 75, 257-268. 
Saalau-Bethell, S., Woodhead, A. J., Chessari, G., Carr, M. G., Coyle, J., Graham, B., Hiscock, S. D., Murray, C. W., Pathuri, P., Rich, S. J., Richardson, C. J., Williams, P. A., Jhoti, H. (2012) Discovery of an allosteric mechanism for the regulation of HCV NS3 protein function. Nature Chemical Biology, 8, 920-925.

Sanders, W. J., Nienaber, V. L., Lerner, C. G., McCall, J. O., Merrick, S. M., Swanson, S. J., Harlan, J. E., Stoll, V. S., Stamper, G. F., Betz, S. F., Condroski, K. R., Meadows, R. P., Severin, J. M., Walter, K. A., Magdalinos, P., Jakob, C. G., Wagner, R., Beutel, B. A. (2004) Discovery of potent inhibitors of dihydroneopterin aldolase using CrystaLEAD high-throughput X-ray crystallographic screening and structure-directed lead optimization. Journal of Medicinal Chemistry, 47, 1709-1718.

Shumlin, I. A., Cymborowski, M., Chertihin, O., Jha, K. N., Herr, J. C., Lesley, S. A., Joachimiak, A., Minor, W. (2012) Identification of unknown protein function using metabolite cocktail screening. Structure, 20, 1715-1725.

Spurlino, J. C. (2011) Fragment screening purely with protein crystallography. Methods in Enzymology, 493, 321-356.

Stout, T. J., Schellenber, U., Santi, D. V., Stroud, R. M. (1998) Crystal structures of a unique thermal-stable thymidylate synthase from Bacillus subtilis. Biochemistry, 37, $14736-14747$.

Tiefenbrunn, T., Forli, S., Happer, M., Gonzalez, A., Tsai, Y., Soltis, M., Elder, J. H., Olson, A. J. Stout, C. D. (2013) Crystallographic fragment-based drug discovery: use of a brominated fragment library targeting HIV protease. Chemical Biology and Drug Design, 83, 141-148.

Verlinde, C. L. M. J., Fan, E., Shibata, S., Zhang, Z., Sun, Z., Deng, W., Ross, J., Kim, J., Xiao, L., Arakaki, T., Bosch, J., Caruthers, J. M., Larson, E. T., LeTrong, I., Napuli, A., Kelley, A., Mueller, N., Zucker, F., Van Voorhis, W. C., Buckner, F. S., Merritt, E. A., Hol, W. G. J. (2009) Fragment-based cocktail crystallography by the medical structural genomics of pathogenic protozoa consortium. Current Topics in Medicinal Chemistry, 9, $1678-1687$.

Verlinde, C., Kim, H., Bernstein, B. E., Mande, S. C., Hol, W. G. J. (1997) Antitrypanosomiasis drug development based on structures of glycolytic enzymes. In Structure-based Drug Design (Veerapandian, P., ed.), pp. 365-394, Marcel Dekker, Inc.

Wang, A., Bolen, D. W. (1997) A naturally occurring protective system in urea-rich cells: Mechanism of osmolyte protection of proteins against urea denaturation. Biochemistry, 36, 9101-9108.

Wilcken, R., Liu, X., Zimmermann, M. O., Rutherford, T. J., Fersht, A. R., Joerger, A. C., Boeckler, F. M. (2012) Halogenated-enriched fragment libraries as leads for drug rescue of mutant p53. Journal of American Chemical Society, 134, 6810-6818. 
Winn, M. D., Ballard, C. C., Cowtan, K. D., Dodson, E. J., Emsley, P., Evans, P. R., et al. (2011) Overview of the CCP4 suite and current developments. Acta Crystallographica Section D Biological Crystallography, 67, 235-242.

Yancey, P. H., Clark, M. E., Hand, S. C., Bowlus, R. D., Somero, G. N. (1982) Living with water stress: evolution of osmolyte systems. Science, 217, 1214-1222.

Yancey, P. H., Somero, G. N. (1979) Counteraction of urea destabilization of protein structure by methylamine osmoregulatory compounds of elasmobranch fishes. Biochemistry Journal, 183, 317-323.

Yuan, P., Bartlam, M., Lou, Z., Chen, S., Zhou, J., He, X., Lv, Z., Ge, R., Li, X., Deng, T., Fodor, E., Rao, Z., and Liu, Y. (2009) Crystal structure of an avian influenza polymerase PA(N) reveals an endonuclease active site. Nature, 458, 909-913.

Zou, Q., Bennion, B. J., Daggett, V., Murphy, K. P. (2002) The molecular mechanism of stabilization of proteins by TMAO and its ability to counteract the effects of urea. Journal of the American Chemical Society, 124, 1192-1202. 


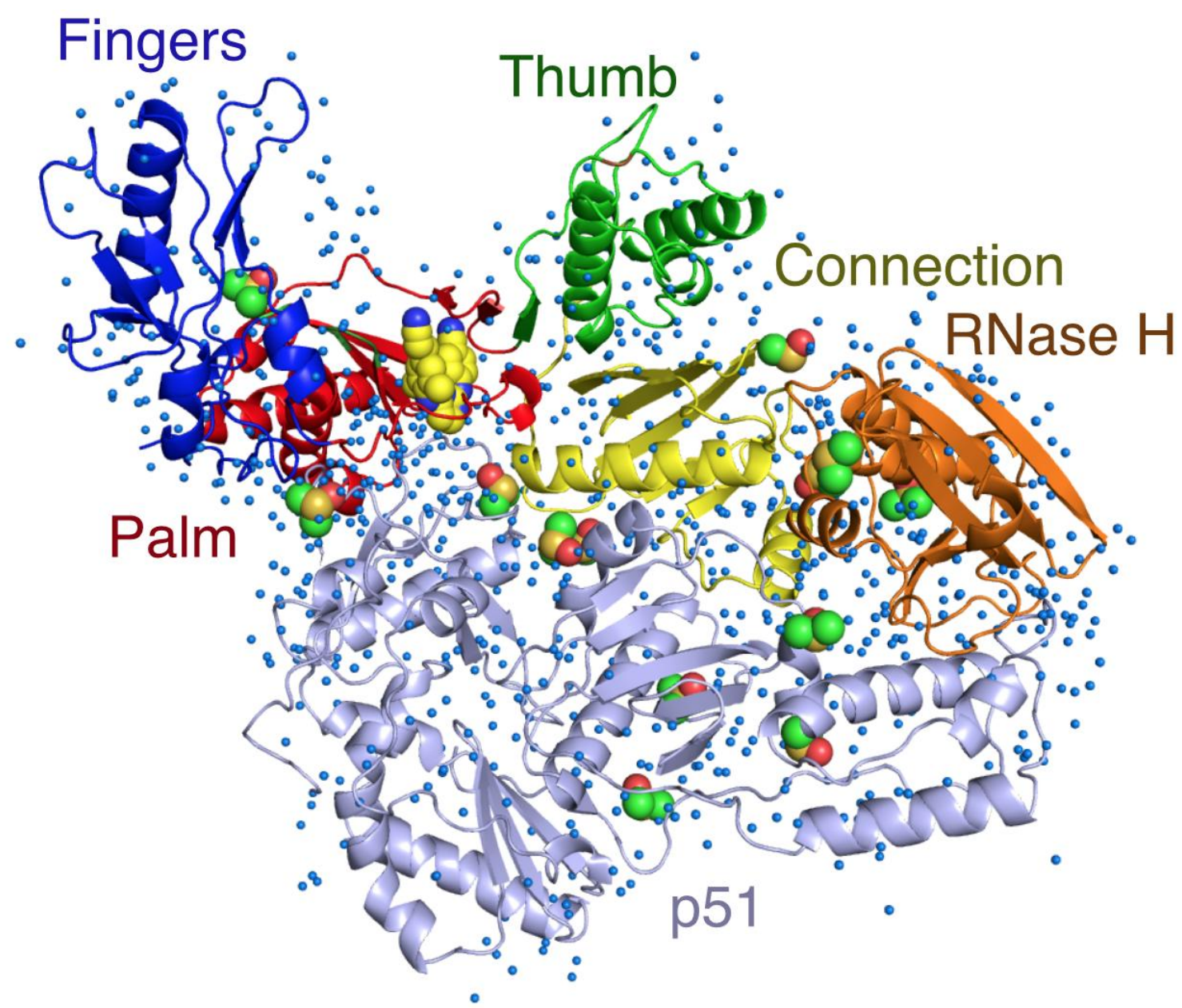

Figure 1: Crystal structure of HIV-1 reverse transcriptase-rilpivirine complex soaked with $20 \%$ (v/v) $\mathrm{d}_{6}$-DMSO. Color-coding: p66 subdomains as fingers (blue), palm (red), thumb (green), connection (yellow), and RNase H (orange), and the p51 subunit (cyan). Rilpivirine is shown as yellow and blue spheres, ordered waters are shown as blue dots, and $\mathrm{d}_{6}$-DMSO molecules are shown as green, yellow, and red spheres. Crystal structure revealed a total of $16 \mathrm{~d}_{6}$-DMSO binding sites. This crystal form was very robust to fragment soaking leading to $\sim 90 \%$ of crystals yielding high-resolution datasets. 


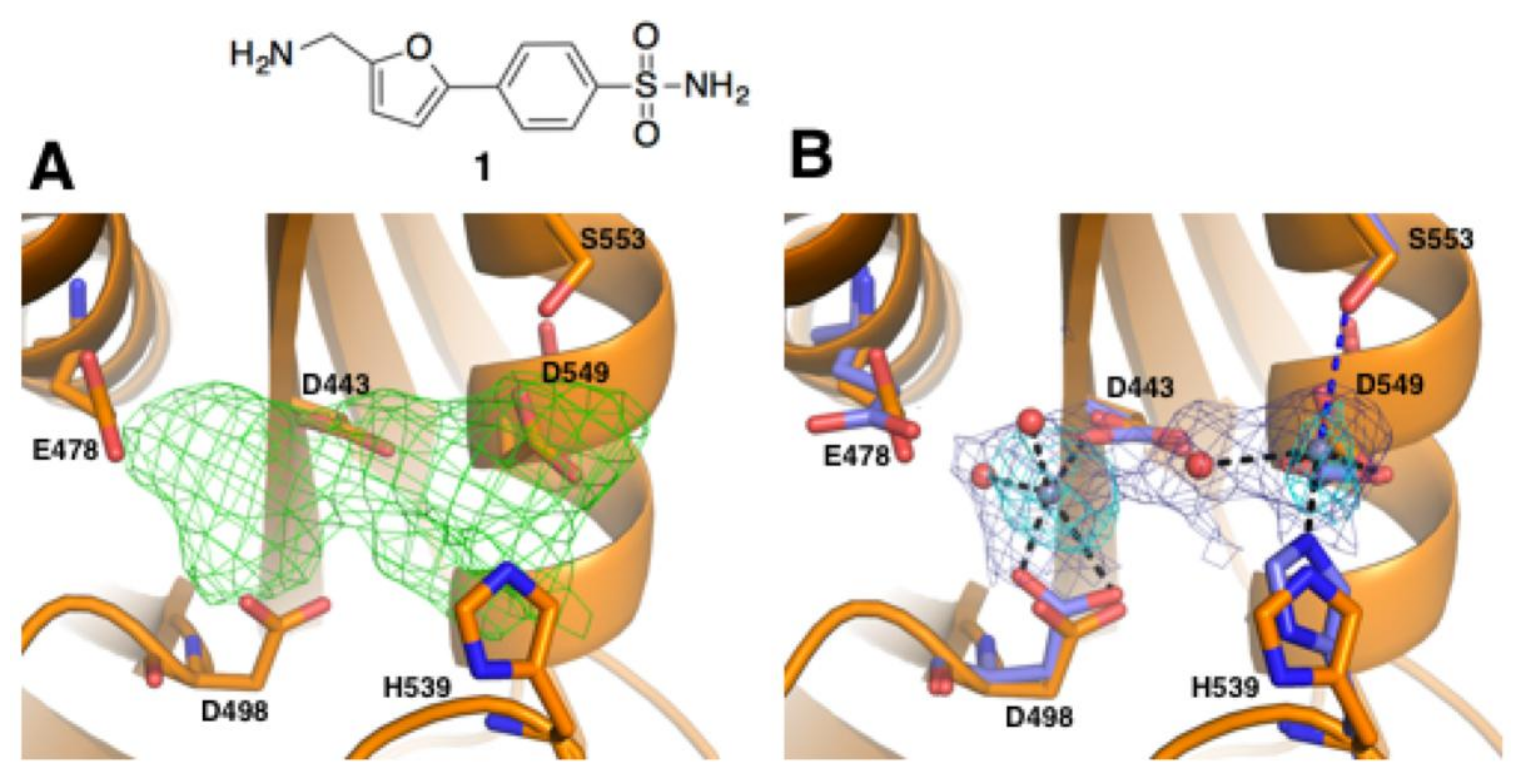

Figure 2: Importance of quality control of compounds used for crystallographic screening. A.) Electron density from a difference map (contoured at 3.0 $\sigma$ ) observed upon soaking of 1 into crystals of HIV-1 RT-rilpivirine complex. Electron density envelope does not accommodate the ligand in question. B.) Electron density with metal ions and coordinating water molecules modeled in. Anomalous density indicating probable metal binding is also shown (cyan mesh contoured at 3.0\%). The metal atoms were unexpectedly present in a "pure" commercial sample; apparent high inhibition (RNase H) led to excitement that was mitigated when fractionation and purification of the compound resulted only in loss of activity.

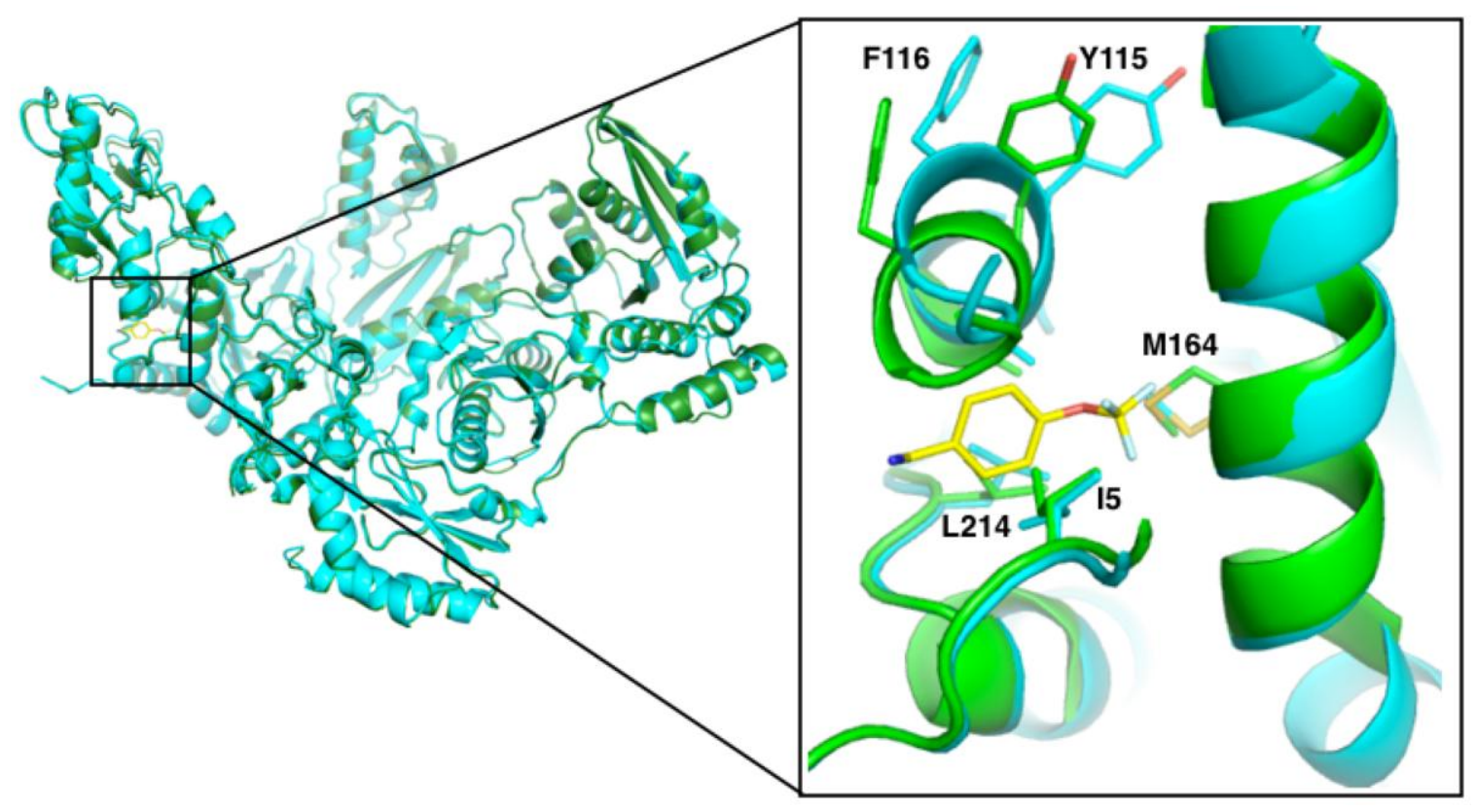


Figure 3: Crystal structure of RT-rilpivirine with a fragment bound at the Knuckles site, revealing a novel allosteric inhibitory site for HIV-1 RT that is potentially druggable.

This pocket is not observed to be open in any of hundreds of published RT structures, nor in the many hundreds of structures solved during our fragment screening campaign, unless occupied by similar fragments. Residues that shift in presence of bound ligand are shown in cyan and in their non-bound positions as green. Y115 and F116 form part of the substrate dNTP-binding site, suggesting that these conformational changes may account for the observed inhibition by fragments binding to this site.

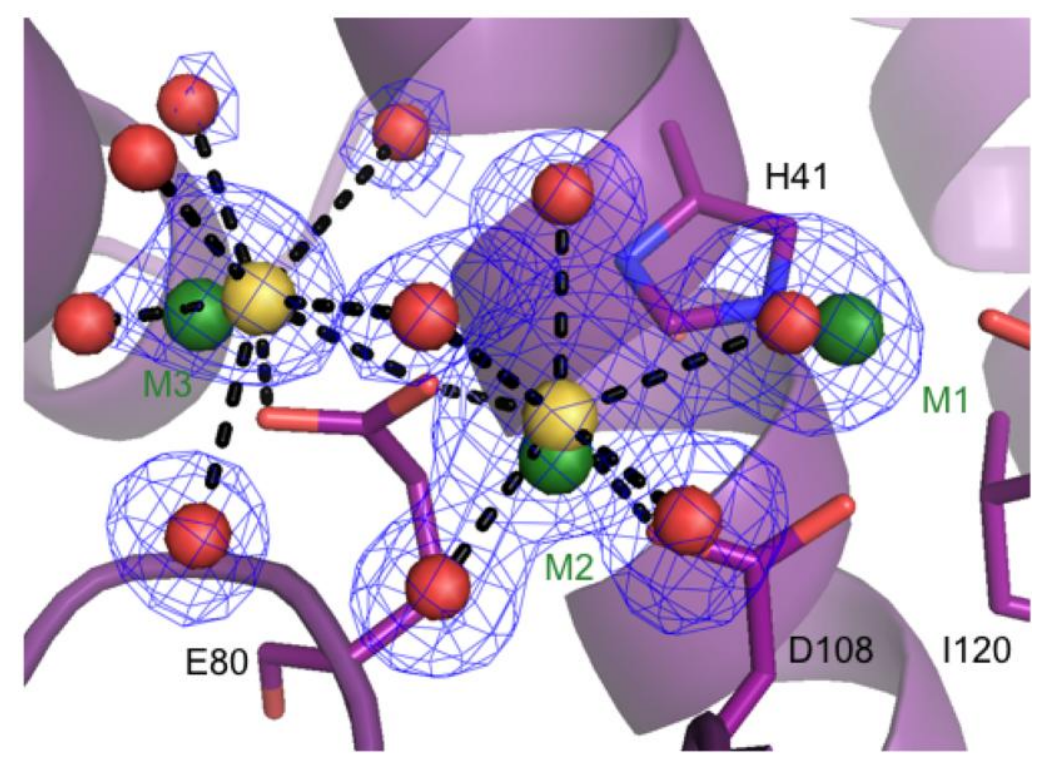

Figure 4: Cartoon representation of apo $\mathrm{PA}_{\mathrm{N}}$ active site from a $100 \mathrm{mM} \mathrm{CaCl} 2$ soak (inspired by observation of a third $\mathrm{Mn}^{2+}$ during fragment screening) showing electron density (light blue mesh contoured at $1.5 \sigma$ ) for the metal ions and coordinating waters. The locations of the metal ions from a fragment soak are displayed in green whereas the locations of the calcium cations from a $100 \mathrm{mM}$ soak are in yellow. The third metal had not been observed in previously reported structures and may have functional significance in stabilization of the reaction products. 


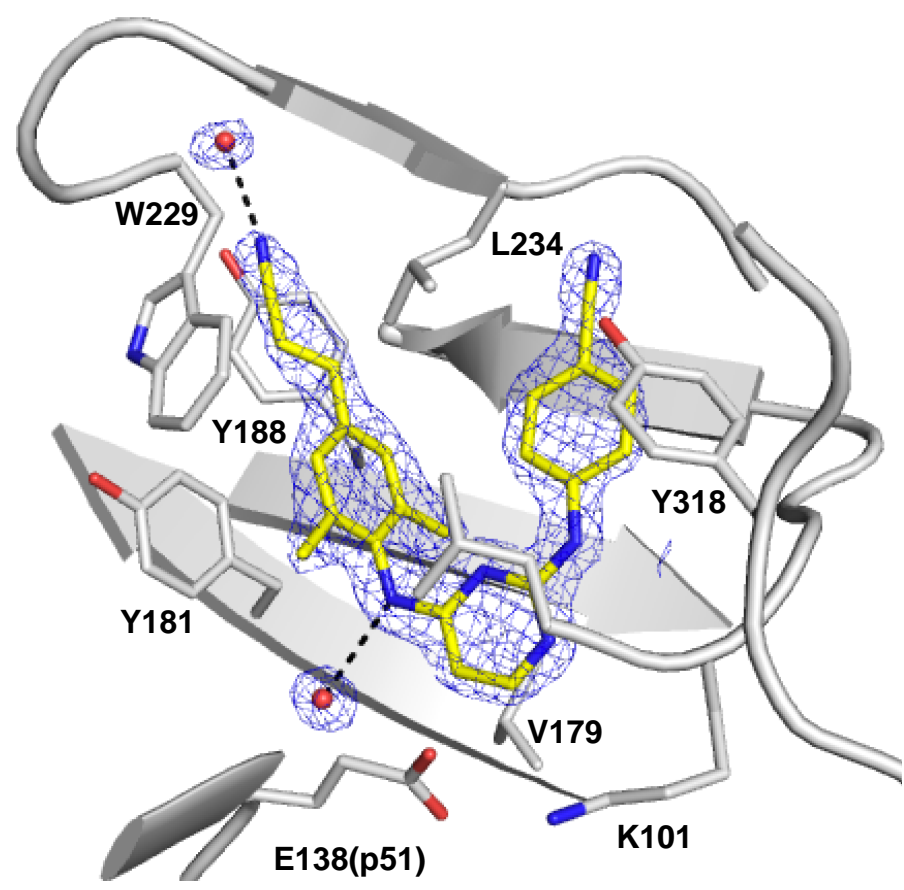

Figure 5: Crystal structure (1.51 ̊ resolution) of HIV-1 RT-rilpivirine complex (RT52A construct), showing two water molecules making hydrogen-bonding interactions (dashed lines) with the bound drug molecule. The previously undetected water molecule (upper left corner) may account for some of the increased inhibitory potency of rilpivirine (in yellow) conferred by the cyanovinyl substituent vs. related diarylpyrimidine analogs. The crystal used for data collection was treated with $6 \%(\mathrm{w} / \mathrm{v})$ TMAO in the cryoprotective solution; without TMAO this crystal form usually yields $1.8 \AA$ resolution datasets. 


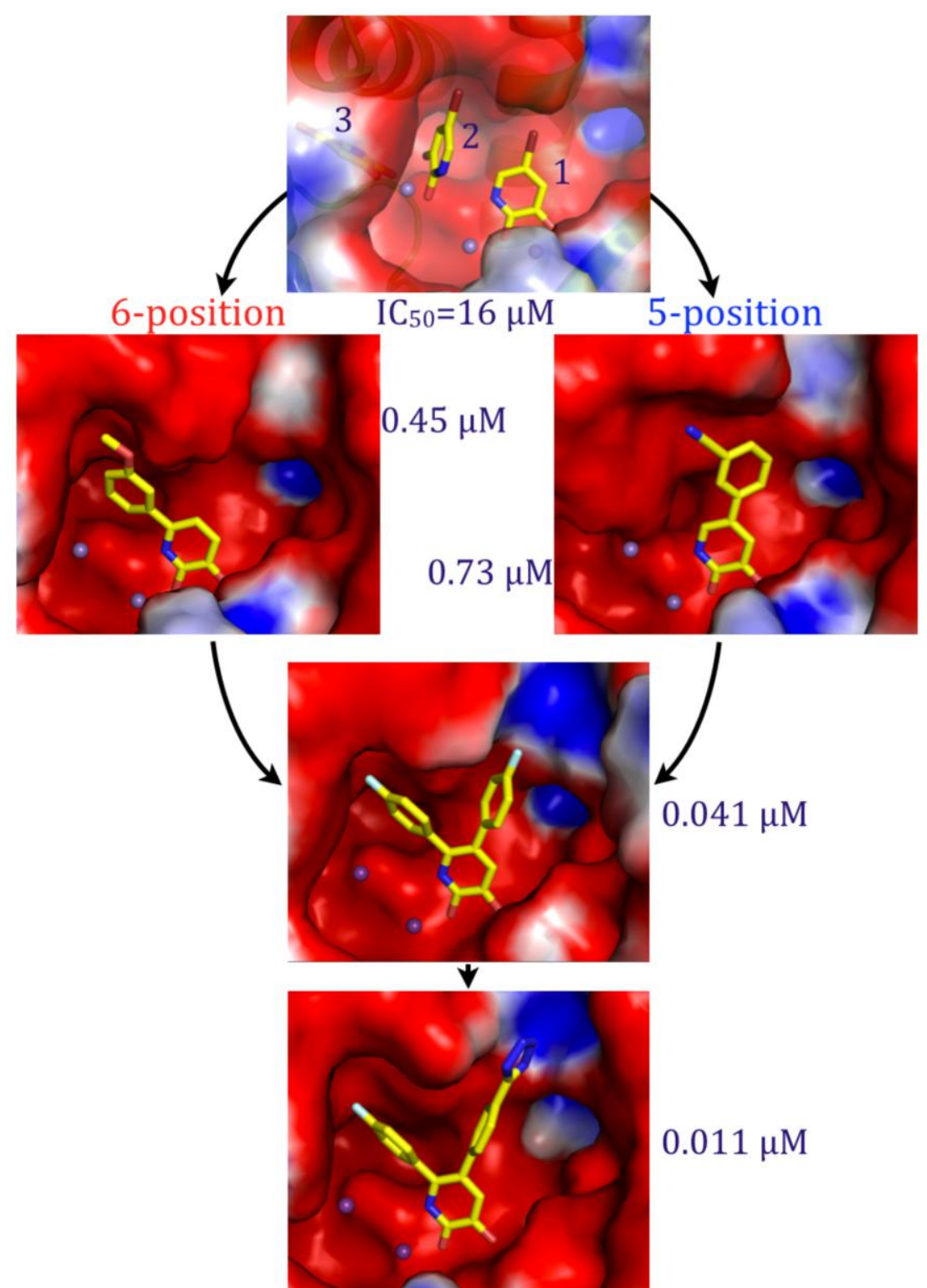

Figure 6: Structure-guided lead development of a fragment hit, 5-chloro-2,3dihydroxypyridine, identified from a screening campaign targeting influenza endonuclease. Electrostatic surface (APBS) is shown for each ligand bound structure. The availability of numerous crystal structures allowed for molecular modeling to be used, resulting in not only an increase in the specificity of binding to the active site but also an improvement in the inhibitory activity of the compounds. Merger of substitutions at the 5- and 6-positions yielded compounds with greater than a thousand-fold increase in potency compared to the initial hit. 